\title{
BUILDING ASSESSMENT USING SHADOW ANALYSIS FOR THE ARCHITECTURAL DOCUMENTATION
}

\author{
Nada Kadhim ${ }^{1 *}$, Nabil Kadhim ${ }^{1}$ \\ ${ }^{1}$ Dept. of Civil Engineering and Engineering Faculty, \\ Diyala School of Engineering, University of Diyala, Baqubah, \\ Diyala, Iraq - (nada.m.kadhim, nabil.ms)@engineering.uodiyala.edu.iq
}

\section{Commission VI, WG VI/4}

KEY WORDS: Architectural Documentation, VHR Satellite Images, Building Detection, Shadows, Partitioning, Labelling, Cultural Heritage

\begin{abstract}
:
One essential approach in preserving architectural heritage is the documentation of building geometries and their sites are thoroughly photographed of existing buildings. For instance, an automated detection of the locations and the extraction of geometry information of the buildings are intrinsic attributes of the architectural design of building interiors and exteriors in cultural heritage documentation and preservation. In this study, contributions of remotely sensed images to detect the sites and shapes of the buildings based on shadow analysis were evaluated. The aim of this paper is to develop an approach to assist in recording and documenting the external geometry of historic buildings and their real location supplying much ease and time-saving in protection projects of cultural heritage. A distinctive merit of the proposed approach is its ability to document the spatial distribution of the detecting buildings by extracting the graphic coordinates of their corners without any additional data. Our experiments demonstrate the efficiency of the proposed approach on two cases, including the architectural documentation of external edges, shape, size, site, orientation, and coordinates. Thus, our new approach offers a tool to document architectural and cultural heritage which can help in capturing and recording all the relevant data and information, both in visual and written format.
\end{abstract}

\section{INTRODUCTION}

The digital documentation of the shape and location of historical and heritage buildings is an indispensable process for enabling long-term archiving, easy sharing, the protection of historic inherited buildings and digital presentation for dissemination and education purposes. Conservation of cultural heritage is a major issue for modern societies in terms of economical and cultural viewpoints. Historic structures and sites are evidence to the ancient civilizations and their historical events in the past (Yilmaz et al., 2007). Cultural Heritage, including archaeological sites, plays a substantial role in: (1) depicting the ancient live of the people, (2) recovering a sense of continuity, dignity and empowerment, (3) supporting the spiritual wellbeing of people, (4) helping attract investments, (5) preserving environmental sustainability and (6) contributing directly to the richness of the living environment for present and future generations (Yilmaz et al., 2007, Xiao et al., 2018). However, every year some of these historic buildings disappear, lost to future generations due to the scourges of wars and insufficient attention to protect these places from the abusers and saboteurs, in particular, developing countries (e.g. Iraq). Additionally, while building geometry information has been recorded for small samples areas, it has not been accurately documented on the scale of entire building surfaces. This is critical, because the exterior appearance of the building's architecture may change or disfigure over time as well as the external edges of the facades and walls of some parts of the building may be exposed to damage. Therefore, the documentation of the external geometries and the sites of historic buildings prior to their exhaustion or sabotage is

\footnotetext{
*Corresponding author: nada.m.kadhim@gmail.com
}

crucial, and could be performed with the assist of remotely sensed images. Satellite images could be useful for locating individual buildings, providing information about their shapes, virtual modelling the environment nearby (Ok, Baseski, 2015). Very High Resolution (VHR) satellite imageries are capable of providing high level of detail because of their ability to offer sub-metre resolution (Kadhim et al., 2015b). This may help services responsible for rescuing and protecting historical buildings. Satellite data is a massive spatial information system that supplies much ease, precision and time-saving in measured drawing projects for cultural heritage documentation and preservation when compared with conventional methods.

This paper is devoted to the automated detection of individual building visible in a single multispectral high resolution satellite image. The motivation to use multispectral images is that the documentation of the cultural heritage places represented by historical buildings (e.g. palaces, heritage buildings, archaeological site and old houses) can be performed by using the analysis of their shadow regions. Presence of the shadow regions can be efficaciously utilised to verify the existence of a building structure because a cast shadow remarkably gives strong evidence of an off-terrain object (Kadhim et al., 2015a). Furthermore, the ability to distinguish shadow regions from non-shadow regions is easier when using near-infrared band (INR) because shadow regions exhibit lower radiance values over the entire spectrum, and due to scattering, shadow regions in sensor irradiance decrease from short to long wavelengths $(\mathrm{Ok}, 2013)$. Therefore, we focus on documenting the external shape (edges) and location of the heritage and historical buildings from satellite images based on shadow regions. Additionally, the use of remotely sensed data can aid us to conduct the documentation and exploration of the cultural 
heritage and archaeology places for analysis, monitoring and management for small-to-medium and large archaeological site scale heritage documentation which can be labour intensive and time-consuming, especially for sites that are unsuitable for in-situ surveying, such as areas of conflict.

Our contribution is to propose a new approach to automatically detect and document buildings from VHR multispectral satellite images. The approach considers the symmetric nature of the cast shadow shape when a well-known building shape (rectangular) intercepts the path of incidence sunlight to extract the building geometry. The proposed approach involves the establishment of two bounding boxes inside and outside the detected shadow regions in which the entire shape is extracted. The calculations also comprise the angle of rotation and the edge alignment of the building to find the drawing coordinates of the corners of a required building.

To remainder of this paper is organised as follows. Section 2 presents the previous studies. The proposed method is given in Section 3. Section 4 reports and discusses the findings. The conclusion and the future directions are provided in Section 5.

\section{PREVIOUS STUDIES}

Automated documentation of archaeological and heritage buildings from images is actively contributing to one or more aspects of heritage conservation. It is usually the fundamental step for assessing the worth of cultural heritage based on various criteria, such as historic, physical, social, architectural and archaeological value is the identification of the location of a heritage site or structure. Although authorities or local people know most of the cultural heritage, much remains hidden or undiscovered. Remote sensing and aerial photogrammetry can be employed for both archaeological site exploration and mapping. They have successfully been utilised to reveal previously unrecognised archaeological heritage. In this context, for instance, seventeen new pyramids in the Nile Valley have recently been discovered using satellite imagery (Parcak et al., 2016). In a different work, (Parcak, Tuttle, 2016) discovered new monumental structures in Jordan using Google Earth, WorldView and Unmanned Aerial Vehicles (UAV) imagery. (Giardino, 2011) displayed the history of NASA remote sensing applications in archaeology. (Kadhim et al., 2016) reviewed remote sensing contributions to urban studies including the use of multispectral and hyperspectral imagery, beside active sensing data, for the discovery, characterization and analysis of building locations worldwide. Through remote sensing data and GIS, (Agapiou et al., 2015) discussed cultural heritage management and monitoring to understand the conservation circumstances of heritage monuments in dangerous conditions and events. (García-Gago et al., 2014) exploited the perspective of the image for the 2D localisation and measurement of a destroyed historic building. In a recent study, (Agapiou, 2017) used Petabyte-scale satellite data which are available on Google's Earth Engine platform for global scale heritage monitoring and management.

According to use shadow information, shadow in the VHR satellite imageries provides vital information on urban construction forms, illumination direction, and the spatial distribution of the objects; all of these can help to further means of documentation tools for assessing and preserving the archaeological cultural heritage. In this regard, many researchers employed the extracted shadow regions from VHR satellite images for different applications. For example, (Redweik et al., 2013) built a shadow algorithm to calculate shadow maps for the solar potential assessment of all surfaces (roofs and facades) of buildings within an urban landscape. (Kadhim , Mourshed, 2018) developed a new algorithm to estimate the height of the buildings based on the extracted shadow areas from VHR multispectral images. (Yan et al., 2010) examined the form shadow and cast shadow changes on the surfaces of architectural heritage buildings to retrieve their original colours for recording and documenting the chromatic information. However, in this aforementioned study, shadow and shade areas were deleted to achieve the documentation of the surface colours of architectural and heritage buildings.

In our work, shadow information is the key input to detect, assess, document geospatial data of the archaeological cultural heritage (e.g. the sites and coordinates of the existence of cultural heritage ), and the representative boundaries of the shadow regions are used to infer the edges of the detected buildings.

\section{METHOD}

The method takes advantage of Very-High-Resolution (VHR) satellite imagery, which can provide important information on the building structures. By detecting and recording the geospatial information (e.g. location and site), in addition to the external geometry information (e.g. shape) using VHR satellite images without any additional auxiliary data, we can achieve more complete documentation for architectural heritage. To this end, we built a new algorithm based on the extraction and analysis of shadow regions employing image processing and computer vision techniques. The geometry including the external edges and real geospatial information of any building from VHR satellite images can help us for the documentation of historical architecture in terms of their shape and site for the wide heritage regions in an effective manner.

An overview of our proposed framework is depicted in Figure 1. The robustness of the proposed approach is to retrieve the shape of buildings automatically by exploiting the shadow areas from VHR satellite images, without having any extra information. Because a cast shadow and its presence in satellite images provide useful information to verify the existence of a building structure with a certain geometric configuration. Shadow regions are distinguished from non-shadow areas based on the characteristics of VHR satellite ortho-images using the bands (green $(\mathrm{G})$, red (R), and near-infrared band (NIR)). Similar to our previous work (Kadhim , Mourshed, 2018), we detected an extracted shadow regions of the existing buildings in the image space. Therefore, in our new method, we produced a binary image by applying Normalized Differential Vegetation Index (NDVI) to detect vegetated areas and the automatic histogram thresholding based on Otsu's method (Otsu, 1979) to compute a binary vegetation mask. A ratio computed with the saturation and intensity components of the Hue-Saturation-Intensity (HSI) space was used as another index to detect shadow regions (Teke et al., 2011). Next, the regions belonging to vegetated areas are subtracted from the whole binary image to obtain a binary shadow mask. We performed a post-processed shadow mask by applying a constrained region growing process to detected shadow regions and a new directional morphological processing to remove the shadow areas corresponding to relatively small objects. The directional spatial relationship between buildings and their shadows has been modelled to detect the accurate 
footprint shape of each building. We, then, achieved a rectangle bounding box surrounding shadow regions to detect and extract the geometry of building footprints (shape and edges) utilising alignment, re-orientation and rotation the angle of the rectangle bounding box. The shape and the coordinates of the corner points for each building within the image were labelled and extracted.

In Figure 1, we present the flowchart of our new algorithm in which its steps can be detailed as follows:

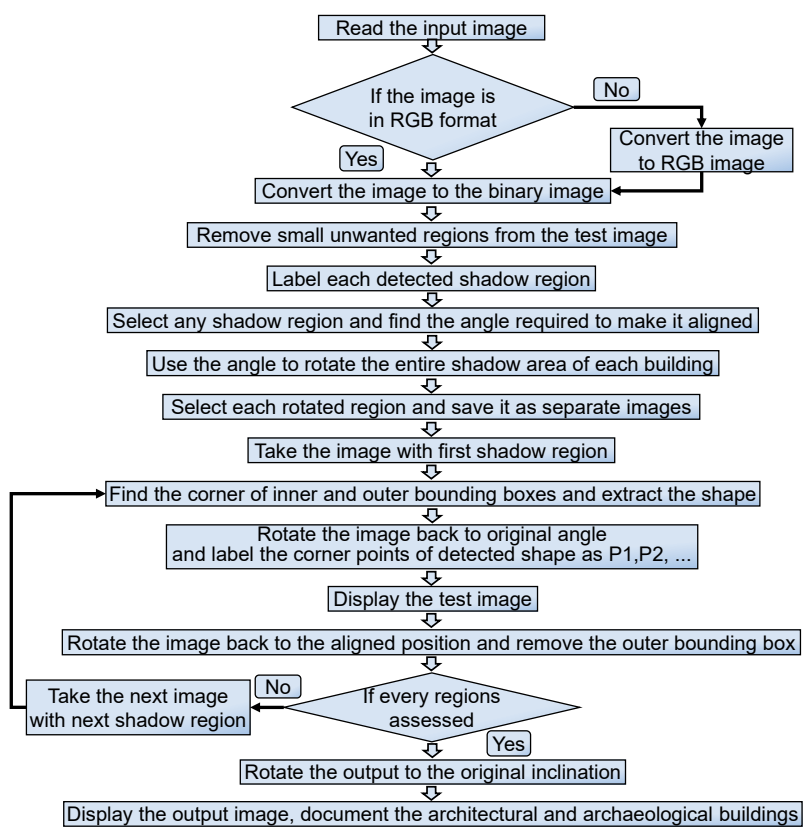

Figure 1. Proposed methodology for the architectural documentation

Step 1: Read the image

Step 2: Resize image and convert the image to 3 bands

2.1. Read the size of the image (no. of rows, columns and no. of bands)

2.2. If the number of bands is not equal to 3 , covert the image to 3-bands

2.3. If the number of rows in the image is less than 300 , resize the image resolution to $150 \%$

Step 3: Remove unwanted objects from the test image

3.1 Define a pixel area 'cut' and used as a threshold to remove small unwanted white objects

3.2 Convert the image to the binary image $\left(I_{1}\right)$

3.3 Remove every small connected regions in the test image $\left(I_{1}\right)$ with areas lesser than 'cut' and saved to $\left(I_{2}\right)$

Step 4: Padding zero values to the image

4.1. Add zero arrays to both rows and columns $\left(I_{3}\right)$

Step 5: Label each white objects with unique values $(L)$ and find the no. of objects (tot)

Step 6: Identify the angle required to make the $L$ - shape white regions (shadow regions) aligned with the horizontal and vertical axes

6.1 Select a white object from the whole image and pass to a function 'calc $c_{a}$ gle'

$$
6.2 \text { for } \mathrm{i}=1 \text { to } 20
$$

Rotate the input image to the $i$ degree white region
Save the values to a matrix $N$

end

6.3 Find the angle which has a maximum $N_{R}$ value and minimum $N_{C}$ value and consider it as the required angle

Step 7: Rotate the ' $L$ ' image to the aligned pose

Step 8: Separate each white object and save it separately to a cell

Step 9: Find the inner and outer bounding box for each object for $\mathrm{i}=1$ : no. of connected regions (shadow pixels)

Load the i white object (shadow region)

Find the leftmost $\left(L_{O}\right)$ and rightmost $\left(R_{O}\right)$ column which includes the shadow pixels

Find the topmost $\left(T_{O}\right)$ and bottom-most $\left(B_{O}\right)$ row which includes the shadow pixels

Create the bounding box in image ' $L$ ' using the above found values. It will be the outer bounding box

The left column and the top row of the inner rectangle will have a property that at column and row, the pixel changes from white to black.

Find the column where white to black transition is maximum and consider it as LeftIn $\left(L_{I}\right)$

Find the row at which the transition from white to black is high and take that value as TopIn $\left(T_{I}\right)$

The bottom row and the right Column of the inner bounding box are taken as same as $B_{O}$ and $R_{O}$ respectively

Create the Inner rectangle in ' $L$ ' using $L_{I}, R_{O}, T_{I}$ and $B_{O}$

Proceed the next steps if the outer bounding box has a minimum area of 25 pixels. This will help to remove very small objects.

Mark the inner bounding box corner pixels in the image by replacing the corner position green band value to 160. (This step is required to identify the corner points after rotating the image to the original position)

Rotate the above image to the original angle position by reversing the rotation using the same angle

Find the corner points by checking the pixels for 160 and insert text labels like $P_{1}, P_{2}, P_{3} \& P_{4}$ if it is the first white object (shadow area)

Thereafter, the image has the original angle with Inner bounding box, outer bounding box and corner labels.

Save the corner values and corner labels to a table to use later

Display the image for 2 seconds

Keep Inner bounding box and its corner points, and then, remove the outer bounding box.

Detect the pixel colour used to create the the outer bounding box and replace those pixels with zero values (The outer bounding box will be removed)

Detect the pixels in the image with object label value and replace it with zeros (Particular, the white object will be removed)

The new output image $(\mathrm{J})$ is used as the input to next iteration. So after executing each iteration, each object will be deleted leaving the corner points and corner labels) end

Step 10: Display the image after completing the step 9 and validate the findings for every extracted building

Step 11: Save the table of the geometric building coordinates to a separate file (a document of site and shape of the heritage buildings) and display it

The iteration of the algorithm performance continues until each building within images space is recorded. 


\section{RESULTS AND DISCUSSION}

\subsection{Image Data Set}

The experiments are performed on two ortho-rectified images acquired from two different satellites, QuickBird $(60 \mathrm{~cm})$ and Geoeye-1 $(50 \mathrm{~cm})$. The two test images comprised four bands $(\mathrm{R}, \mathrm{G}, \mathrm{B}$, and NIR) and three bands (R, G and $\mathrm{B})$ in the QuickBird image and the Geoeye-1 image, respectively, with a radiometric resolution of 11 bits per band.

\subsection{The Outputs of The Developed Algorithm}

The findings are promising for recording and documenting the geometry of historic buildings and their sites for conducting further protection processes and spatial analysis. While the algorithm helps identify regions of buildings, their geometry, orientation, and location relating to other buildings it is not intended to solve the problems of the internal geometry of the historic buildings or the accurate identification of the architectural design of the buildings. Instead, it provides documentation for existing building shapes including their attributes (e.g. edges and dimensions), and has the potential to help monitor the fading process, and assist in the restoration of documented geometric shapes.

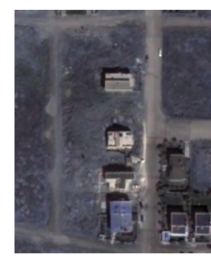

(a)

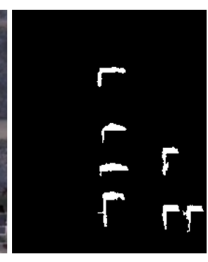

(b)

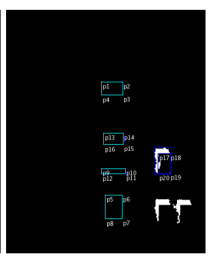

(c)

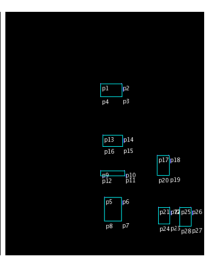

(d)
Figure 2. The implementation of the developed algorithm for architectural documentation. First test image, (a) QuickBird pan-sharped image (R,G, B and INR), (b) the extracted shadow regions after removing the small unwanted areas, (c) the extraction of the building geometry, (d) the location and shape of the detected buildings

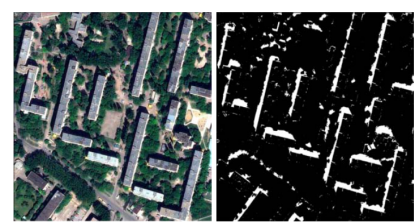

(a)

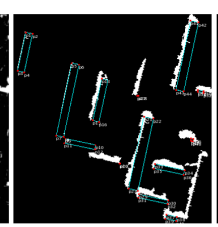

(c)

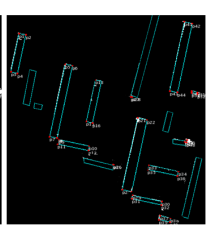

(d)
Figure 3. The implementation of the developed algorithm for architectural documentation. First test image, (a) Geoeye-1 pan-sharped image (RGB), (b) the extracted shadow regions before removing the small unwanted areas (here, we provided two cases to present the binary image of the detected shadow regions with and without the existence of unwanted areas), (c) the extraction of the building geometry, (d) the location and shape of the detected buildings

The developed algorithm was coded in MATLAB, and it was applied to a single VHR pan-sharpened multispectral satellite images. Although we were not able to apply our algorithm to the actual heritage buildings, the developed algorithm can be used to detect, assess and document the geometry of any specific building, and determine its real spatial location, as shown in Figure 1 and Figure 2. It was difficult to obtain VHR satellite images presenting cultural heritage sites when this study was achieved but the test images that we used displayed the ability to identify the existence of buildings from their shadow regions despite the complex urban landscape.

In this study, we consider the task of the documentation of the buildings as a binary partitioning problem where a given building region has to be accurately separated from its background. We extracted the location of the buildings based on detecting the shadow regions of the apparent buildings in image space. The detection and extraction of the shadow regions were explained in details by (Kadhim et al., 2015b). Since the proposed approach depends on shadow information, the two test images are carefully selected to cover varying illumination and acquisition conditions that might be encountered during the use of satellite images for various cultural heritage documentation purposes. In this vein, Figure 1 and Figure 2 illustrate the well-performed work of our new approach of architectural documentation of the existing buildings using VHR multispectral satellite images. However, some buildings, as shown in Figure 2, were not detected well due to the dense vegetation cover. Some cast shadow regions of vegetation combined with shadow regions of the buildings can cause this confusion in the detection process. This occurs when vegetation is adjacent to buildings. (a)

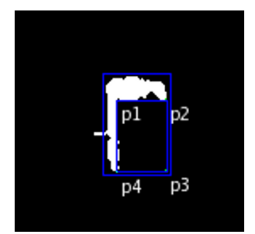

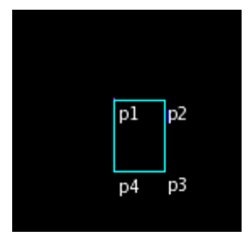

(b)

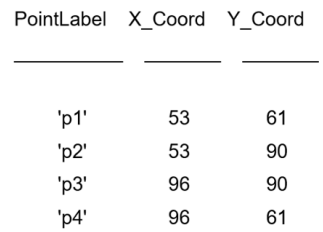

(c)
Figure 4. The documentation of the buildings. (a) the creation of the two bounding boxes (inner and outer), (b) the identification of the building edges (shape), and (c) the extraction of the graphic coordinates of the building corners

The results prove that the proposed approach is quite convincing and remarkable for assessing the entire shape of the detected buildings, and it is capable of extracting and recording the graphic coordinates of the geometry of the buildings and their external edges. The performance of the proposed architectural building documentation approach is evaluated by comparing the results with the reference data. The reference building coordinates for each image are produced manually by a qualified human operator. The extraction of the corners coordinates for each building in the test images gives a strong impression that the developed method is well-performed and exemplary. Nevertheless, not all the extraction coordinates of the corners of the detected buildings were accurate and had the same positions in the reference images because many corners were not extracted in an appropriate manner. The displacement between the coordinates of the same corner point in both the reference and test images belongs to the building edges are not utterly met each other at that corner due to the process of morphological dilation and erosion that conducted on the stage of the shadow detection in which may affect on losing a part 
of the edge. We assessed the algorithm performance of the proposed approach by employing the well-known three metrics (Precision, Recall, $F_{1}$ ) to evaluate the pixel-based performance of the proposed approach as applied in our previous work (Kadhim , Mourshed, 2018)

$$
\begin{gathered}
\text { Precision }=\frac{|T P|}{|T P|+|F P|} \\
\text { Recall }=\frac{|T P|}{|T P|+|F N|} \\
F_{1}=\frac{2 * \text { precision } * \text { recall }}{\text { precision }+ \text { recall }}
\end{gathered}
$$

where $T P$ are the true positives, $F P$ are false positives, and $F N$ are false negatives. The operation $|*|$ denotes the number of pixels assigned to each distinct category. The $F_{1}$ considers both the Precision and the Recall of the test to compute the score, which can be interpreted as a weighted average of the Precision and Recall, where the $F_{1}$ score reaches its best value at 1 and worst at zero.

In the pixel-based performance of the proposed approach, the $F_{1}$ score of approximately $76.75 \%$ was achieved from applying the proposed approach to QuickBird image, and $82.9 \%$ when it applied to Geoeye-1. In the object-based evaluation, the $F_{1}$ score of approximately $89 \%$ and $87 \%$ from applying the method to QuickBird and Geoeye-1 images, respectively.

Based on those visual and numerical assessments, we can conclude that the proposed approach works fairly well and satisfactory for such two diverse and challenging test images.

\section{CONCLUSION}

In this paper, we developed a new approach for the automated documentation of architectural buildings from monocular VHR optical satellite images. Two experiments performed on QuickBird and Geoeye-1 VHR images showed that the proposed approach could fairly localise, detect and record buildings with shapes and sizes in complex environments. We used our previous work to detect and extract shadow regions of the buildings from images. Thereafter, we removed unwanted shadow areas. Next, each region of shadows treated separately through the automated creation of two bounding boxes (inner and outer shadow area). The geometry of each building and its corner coordinates were determined and registered. The tests revealed that the proposed approach could achieve reasonable performance in building detection even under challenging environmental and lighting conditions. In addition, the framework is time-efficient and during the building detection process, user intervention is not required. Our new approach offers a tool to document architectural and cultural heritage which can help in capturing and recording all the relevant data and information, both in visual and written format. Additionally, the use of remotely sensed data can aid us to conduct the documentation and exploration of the cultural heritage and archaeology places for analysis, monitoring and management for small-to-medium and large archaeological site scale heritage documentation which can be labour intensive and time-consuming, especially for sites that are unsuitable for in-situ surveying, such as areas of conflict.
For future work, we plan to develop and integrate a different method that is particularly designed for extracting real coordinates and matching them with the extracted graphic coordinates to obtain the actual building located in the real world. In addition, it is planned to apply and examine our approach to many different satellite images of various historical sites and heritage buildings in Iraq for documenting their locations. Furthermore, the detection results can be further improved by improving the boundaries of the detected building regions by means of an automated creation process of a 3D model for each historic building or monument.

\section{ACKNOWLEDGMENT}

The images utilised in this study were provided by Dr Ali Ozgun Ok. The authors would like to thank him for sharing VHR satellite images with researchers. The authors are also grateful to two anonymous reviewers and the associate editor for their helpful comments.

\section{REFERENCES}

Agapiou, Athos, 2017. Remote sensing heritage in a petabyte-scale: satellite data and heritage Earth Engine(C) applications. International Journal of Digital Earth, 10, 85-102.

Agapiou, Athos, Lysandrou, Vasiliki, Alexakis, Dimitrios D, Themistocleous, Kyriakos, Cuca, Branka, Argyriou, A, Sarris, Apostolos, Hadjimitsis, Diofantos G, 2015. Cultural heritage management and monitoring using remote sensing data and GIS: The case study of Paphos area, Cyprus. Computers, Environment and Urban Systems, 54, 230-239.

García-Gago, Jesús, González-Aguilera, Diego, Gómez-Lahoz, Javier, José-Alonso, San et al., 2014. A photogrammetric and computer vision-based approach for automated 3D architectural modeling and its typological analysis. Remote Sensing, 6, 5671-5691.

Giardino, Marco J, 2011. A history of NASA remote sensing contributions to archaeology. Journal of Archaeological Science, 38, 2003-2009.

Kadhim, Nada, Mourshed, Monjur, 2018. A shadow-overlapping algorithm for estimating building heights from VHR satellite images. IEEE Geoscience and Remote Sensing Letters, 15, 8-12.

Kadhim, Nada, Mourshed, Monjur, Bray, Michaela, 2015a. Automatic extraction of urban structures based on shadow information from satellite imagery. Proceedings of BS2015: 14th Conference of International Building Performance Simulation Association, International Building Performance Simulation Association, 2607-2614.

Kadhim, Nada, Mourshed, Monjur, Bray, Michaela, 2015b. Shadow detection from very high resolution satellite image using grabcut segmentation and ratio-band algorithms. Int. Arch. Photogramm. Remote Sens. Spatial Inf Sci, XL-3/W2, International Society for Photogrammetry and Remote Sensing, 59-101.

Kadhim, Nada, Mourshed, Monjur, Bray, Michaela, 2016. Advances in remote sensing applications for urban sustainability. Euro-Mediterranean Journal for Environmental Integration, $1,7$. 
Ok, Ali Ozgun, 2013. Automated detection of buildings from single VHR multispectral images using shadow information and graph cuts. ISPRS journal of photogrammetry and remote sensing, 86, 21-40.

Ok, Ali Ozgun, Baseski, E, 2015. Automated detection of oil depots from high resolution images: A new perspective. ISPRS Ann. Photogramm. Remote Sens. Spatial Inf. Sci., 1, 149-156.

Otsu, Nobuyuki, 1979. A threshold selection method from gray-level histograms. IEEE transactions on systems, man, and cybernetics, 9, 62-66.

Parcak, Sarah, Gathings, David, Childs, Chase, Mumford, Greg, Cline, Eric, 2016. Satellite evidence of archaeological site looting in Egypt: 2002-2013. Antiquity, 90, 188-205.

Parcak, Sarah, Tuttle, Christopher A., 2016. Hiding in Plain Sight: The Discovery of a New Monumental Structure at Petra, Jordan, Using WorldView-1 and WorldView-2 Satellite Imagery. Bulletin of the American Schools of Oriental Research, 375, 35-51.

Redweik, P, Catita, Cristina, Brito, Miguel, 2013. Solar energy potential on roofs and facades in an urban landscape. Solar Energy, 97, 332-341.

Teke, Mustafa, Başeski, Emre, Ok, Ali Özgün, Yüksel, Barış, Şenaras, Çağlar, 2011. Multi-spectral false color shadow detection. ISPRS Conference on Photogrammetric Image Analysis, Springer, 109-119.

Xiao, Wen, Mills, Jon, Guidi, Gabriele, Rodríguez-Gonzálvez, Pablo, Barsanti, Sara Gonizzi, González-Aguilera, Diego, 2018. Geoinformatics for the conservation and promotion of cultural heritage in support of the UN Sustainable Development Goals. ISPRS journal of photogrammetry and remote sensing, 142, 389-406.

Yan, Wei, Behera, Ajit, Rajan, Pankaj, 2010. Recording and documenting the chromatic information of architectural heritage. Journal of Cultural Heritage, 11, 438-451.

Yilmaz, Haci Murat, Yakar, Murat, Gulec, Saadet Armagan, Dulgerler, O Nuri, 2007. Importance of digital close-range photogrammetry in documentation of cultural heritage. Journal of Cultural Heritage, 8, 428-433. 